\title{
Real Time on Bed Medical Services : A Technological Gift to the Society
}

\author{
Girish Talmale ${ }^{*}$ and Urmila Shrawankar ${ }^{2}$ \\ ${ }^{1}$ Research Scholar, Department of Computer Science and Engineering, \\ ${ }^{2}$ Associate Professor, Department of Computer Science and Engineering, \\ ${ }^{1,2} \mathrm{G}$ H Raisoni College of Engineering, Nagpur (MS), India
}

\section{ABSTRACT}

Nowadays due to the emergence of pandemic diseases like Covid-19, chronic and contagious diseases where remote patient monitoring plays an important role which protect doctors and other healthcare workers from getting contaminated. In India 70\% of the population lives in rural areas where expert medical services are not provided with affordable prices in real time. The existing system is not able to provide real time Medicare services to remote patients. There is a requirement of real time remote prioritization of medical services for remote patients when there is critical change in health parameters. This research presents the real time computing framework for on bed medical services for patients. The real time processing done at the sensor node using real time tasks scheduling. The sensor node data gets analyzed on fog nodes taking time critical actions. The healthcare data is then sent using Kafka real time streaming using Kafka pipeline. The real time processing and analytics of large scale healthcare cloud data using Spark. The proposed framework provides the following benefits over existing systems a) Real time processing of sensory healthcare data b) Real time analytics using fog computing for quick action in critical situations c) Real time data streaming using Kafka pipeline and d) Spark for large scale real time data processing and analytics. The proposed system reduce the requirement of medical resources, workload of medical staff, medical expenses for poorly people and mortality rate of patients with pandemic and chronic disease.

KEY WORDS: REAL TIME SYSTEM, FOG COMPUTING, KAFKA, SPARK, COVID-19

\section{INTRODUCTION}

Rice As of now, the world is confronting the most genuine worries in human services including the ascent of human services costs, the development of the maturing population and the rise of new chronic and pandemic diseases such as Covid-19. Recent advancement in computation and communication technologies plays an important role

\section{ARTICLE INFORMATION}

*Corresponding Author: girish.talmale@raisoni.net

Received 14th Oct 2020 Accepted after revision 29th Dec 2020

Print ISSN: 0974-6455 Online ISSN: 2321-4007 CODEN: BBRCBA

Thomson Reuters ISI Web of Science Clarivate Analytics USA and Crossref Indexed Journal

\section{1) Clarivate $\begin{gathered}\text { Analytics } \\ \text { Crossef }\end{gathered}$}

NAAS Journal Score 2020 (4.31)

A Society of Science and Nature Publication,

Bhopal India 2020. All rights reserved.

Online Contents Available at: http//www.bbrc.in/

Doi: $h t t p: / / d x$.doi.org/10.21786/bbrc/13.14/32 in improving Medical services. In A country like India where $70 \%$ of the population lives in rural areas, it is very difficult to provide expert medical services with affordable prices. Real time on bed patients monitoring systems is the only solution to handle the pandemic diseases like Covid-19. Elderly patients with chronic disease, patients with disability and premature children's require on bed medical services and real time action when there is change in critical health parameters.

The healthcare resources and medical staff in India are limited to handle the pandemic disease like Covid-19 and also there is huge possibility of spreading infections of such disease to doctors and healthcare staff and patient's relative during treatment Implementation of ob bed medical service is the only situation to handle such a pandemic and chronic disease [K. I. Mohammed 2019]. The on bed medical service provides many advantages such as

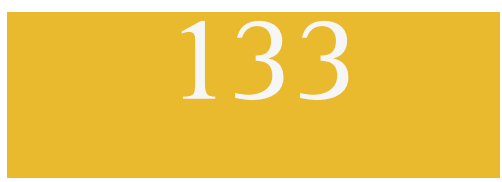


continuous monitoring of health parameters, proactive way to detect and treat illness in real time, prevention from worseness of diseases, reduce the hospitalization fee, real time health parameter monitoring with daily life activities, patients with disability and mobility issues [Iqbal 2019].

Background Work: Existing solutions use telemedicine in which medical information is delivered to remote locations using electronic communication but this system fails to provide real time medical service in case of emergency. Develop mobile healthcare monitoring system to monitor [Hussain 2015] the patient's heart rate using mobile. The drawbacks of this system are to focus on only one parameter and not provide real time response in critical condition. The virtual reality based elderly patients monitoring system with wireless sensor area network for communication which face challenges such as low battery issue, security and less communication speed [Okura 2016] . An e-health system which used Wireless Body Area Network with dynamic priority based architecture which also faced the problem of real time communication [S. Gambhir 2016].Erden in 2016 discussed remote health monitoring using image and signal processing.

Figure 1: Real Time Healthcare Monitoring System [Gu L 2017]

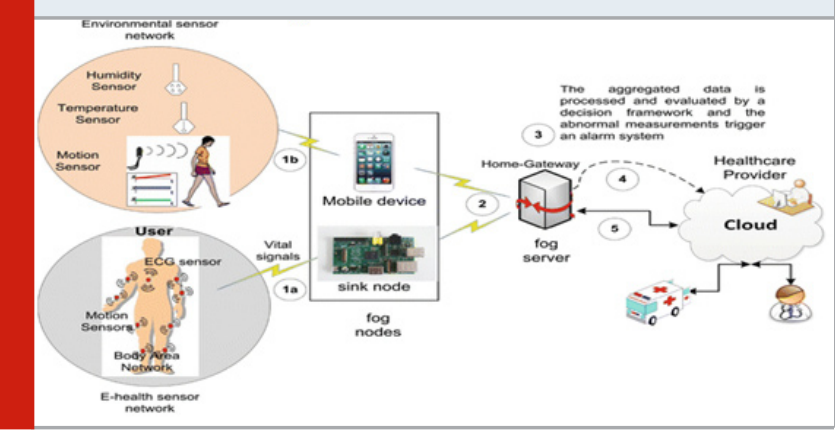

The limitation of this research is not providing real time response systems as it uses cloud based solutions. Abdulnabi presents distributed architecture for medical data exchange and the wireless based patient monitoring system which is used to detect the patient's respiration and sensors to detect coughing [Abdulnabi 2017]. Zhanjal present medicine remainder and monitoring system using IoT in 2015 [Zanjal 2016].The proposed research work presents the real time system framework for on bed medical services which used different sensors like Heart Beat sensor, Glucose sensor, oximeter, Asthma Tracker, Temperature sensor, Camera, Bp sensor, Microphone for cough monitoring, motion sensor, humidity sensor, ECG sensor etc. is used to collect healthcare parameter from patients and process the data in real time using fog nodes on which real time scheduler is used to priorities the the tasks based on criticality of vital healthcare parameters. The sensor data is then first analyze by the fog server and real time quick action or alert is actuate to protect from health hazards. The fog server further send the data to cloud server using real time Kafka data streaming pipeline and Spark is used to process and analyze that large scale healthcare data in real time and provide it to healthcare staff and other government agencies to take further actions as shown in fig.1.

The main objectives of this paper are as follows:

1. To provide real time framework for healthcare monitoring.

2. To implement real time sensory data acquisition system using real time scheduling of critical tasks.

3. Real time data analytics for healthcare monitoring using fog computing.

4. Real time healthcare data streaming using Kafka pipeline.

5. Spark based real time analysis of healthcare data on cloud.

\section{METHODOLOGY}

Real time framework for healthcare system is as shown in Fig.1 where different types of sensors used to collect health information from patients suffering from pandemic and chronic disease, elderly, premature babies . The sensory healthcare data is processed in real time on fog nodes using real time scheduler to priorities the critical tasks and send the real time healthcare information on fog server. Fog server analyzes the data in real time and takes the quick action in real time based on criticality of data. The fog server further stored data on cloud server using real time Kafka streaming pipeline. Spark processes and analyze the large scale healthcare data and provide the analysis to doctors and medical staffs and government agencies as shown in Fig.2.

Real Time Sensing for Healthcare Monitoring: Real time framework provides various healthcares and medical service which helps to take corrective actions in real time. The summary of such application and different types of sensor used for collection of data for these applications is mentioned in the table 1 as follows [A. Botta 2016].

Fog Computing for Real Time Healthcare System: Sensors collect information from patients and send the sensor information to fog nodes for real time analysis . Embedded microcontroller gets information from sensor and processes the real time sensor data to fog nodes. Medical applications are different from other cyber physical system in which remote monitoring requires reliability and security and all operations must be performed in real time which is not possible with cloud computing.

So to implement real time analytics for time critical application such as healthcare fog computing plays an important role. Fog computing in real time health care system provide following advantages [Rahul Neware 2020].

1. Fog computing architecture is distributed which helps to provide reliability to healthcare application

2. Communication is directly with edge devices to provide real time communication service for healthcare application

3. Data processing in fog computing is done very close 
to source of information to take quick corrective action.

4. Large nodes can be connected in fog computing which helps in formation of cyber physical system for healthcare system

5. Fog computing provides less latency require for real time analysis of healthcare system

6. Fog computing provide various protocol standards and higher security as compare to cloud computing.

Figure 2: Real Time Framework for Healthcare Monitoring

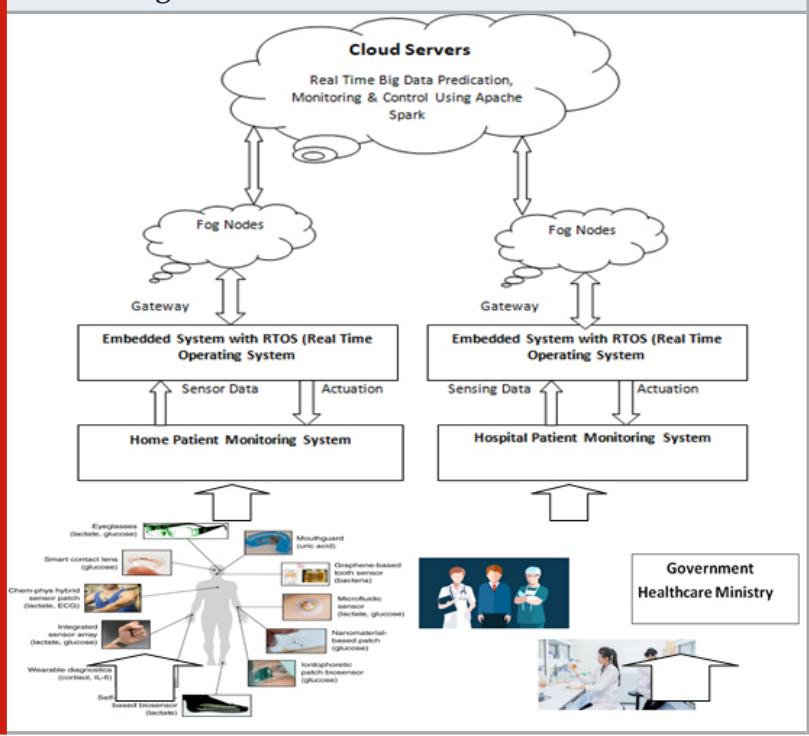

Kafka for Real Time Healthcare Data Streaming: In real time healthcare system can be many to one or many to many system where many sensors sending messages at regular interval. Kafka is a pipeline architecture used to store large amount of data to database on real time. It can be configured as many to many and many to one mode for real time healthcare system. The Kafka is configured and runs using Zookeeper server. The pipeline is created using Kafka topic. When Kafka topic created and it is in running mode then any number of sensor nodes can send data to Kafka topic and any number of actuator nodes can read data from Kafka broker [Kreps, Narkhede \& Rao 2011]. Kafka has the ability to store data for some time in buffer storage and processes the data in real time within the pipeline itself. The cyber physical real time healthcare system architecture consists of sensor within cluster send messages over MQTT cluster for communication. Sensor nodes inside the cluster send data to gateway. Gateway is used to write data to Kafka topic. Kafka pipeline act as real time injection layer that inject data into the HDFS ( Hadoop Distributed File System ) as show in fig.3.

Spark For Real Time Big Data Processing of Health Data: Healthcare data analysis used machine learning algorithms. Existing hadoop system to handle machine learning algorithm has some practical difficulties. Machine learning and deep learning algorithms take long time to produce output. It is executed in multiple stages using MapReduce program in back end [R. Singhal and P. Singh 2014].

Table 1. Real Time Sensing for on Bed Healthcare System

\begin{tabular}{|c|c|c|}
\hline Sr.No & Healthcare Application & Sensor and Operations \\
\hline 01 & Monitoring and Control of Heart rate & Heartbeat sensor is used to track changes in volume of blood in body. \\
\hline 02 & Diabetic Patient Monitoring & Glucose Sensor is used to monitor level of sugar and real time alert \\
\hline 03 & $\begin{array}{c}\text { Asthma Patient Monitoring } \\
\text { updates the real time information to cloud }\end{array}$ & Asthma Tracker is used to track the patient asthma level and \\
\hline 04 & Pulse Rate Monitoring & i0ximeter is used to monitor pulse rate \\
\hline 05 & Temperature Monitoring System & $\begin{array}{l}\text { Temperature sensor used to monitor temperature of patients and } \\
\text { store in information the cloud }\end{array}$ \\
\hline 06 & Blood Pressure Monitoring & BP sensor is used to monitor the real time value of blood pressure \\
\hline 07 & Cough Monitoring system & Microphone used track the real time cough analysis. \\
\hline
\end{tabular}

The problem with map reduce is that it loads the data into memory, processes the data and then store it back. The read and write operation on memory take huge time as comparing to processes data. In healthcare application data must be processes in real time to take corrective action immediately to avoid any risk. Spark is new framework for replacement of MapReduce framework in Hadoop2.0. Table 1.2 shows the difference between spark and hadoop map reduce based on different parameters [M. Armbrust 2015].The spark uses resilient distributed dataset (RDD) and directed acyclic graph.
Figure 3: Kafka Based Data Pipeline Architecture for Real Time Data Streaming

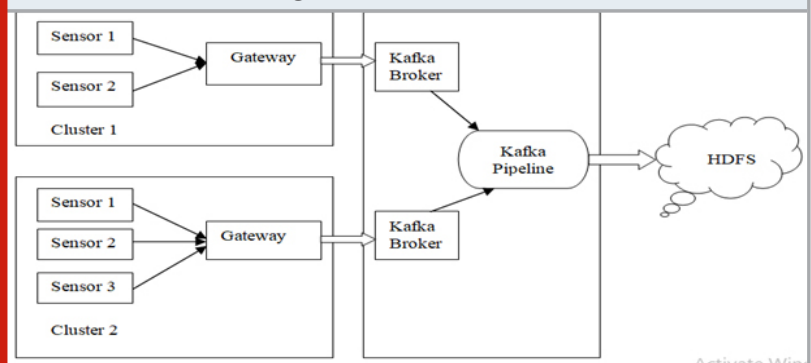


The Spark application read data from used code and placed it into the RDD. The RDD are not changeable and each step it creates new RDD [Mohsin 2018]. The conversion processes is designated as directed acyclic graph and submitted to cluster. The conversion processes consist of arithmetic, logical and set or map based operation. After conversion process RDD get shuffle, then combined and final result get produced. The advantages of using Spark framework in cyber physical healthcare real time system is shown in directed acyclic graph execution step. Directed acyclic graph used end to end processes in that first data get read, transformed and then stored in database but in MapReduce there is memory write operation for each transformation of job.
Figure 4: Spark Framework Architecture for Handling Real Time Processes

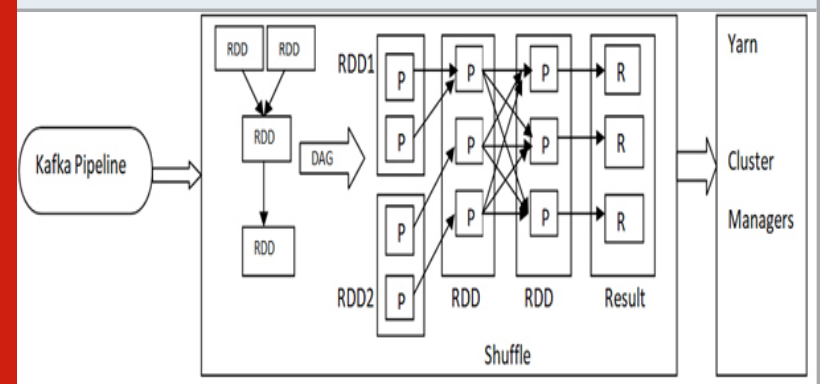

Table 2. Comparative Results of Proposed Real Time on Bed Medical Service Framework

\begin{tabular}{|l|c|c|c|}
\hline Sr.No & Performance Attributes & Existing Systems & Proposed System \\
\hline 01 & Automatic & Semi Automatic & Full Automatic \\
\hline 02 & Real Time & Not Real Time & Real Time \\
\hline 03 & Maintenance of Healthcare Data & Difficult & Easy \\
\hline 04 & Analysis of Healthcare Data & Not Efficient & Efficient \\
\hline 05 & Real Time Prioritization of Healthcare Task & No & Yes \\
\hline 06 & Error Rate & More & Less \\
\hline 07 & Fault Tolerant & No & Yes \\
\hline 08 & Real Time Processing in Fog Node & No & Yes \\
\hline 09 & Real Time Analysis on Fog Server & No & Yes \\
\hline 10 & Real Time Data Streaming & No & Yes \\
\hline 11 & Analysis of large scale data using & No & Yes \\
\hline 12 & Data Analysis at Fog Server & No & Yes \\
\hline 13 & Security & Less & High \\
\hline
\end{tabular}

Yarn is used as cluster management of tasks to produce the result. The Spark framework store intermediate result into another RDD and combine it during processing which reduce the time require for execution depending upon the job and machine learning or deep learning model as shown in fig.4.

\section{RESULTS AND DISCUSSION}

The real time framework for on bed medical services provides several benefits over existing remote patients monitoring systems. The table 2 represents the comparative analysis of existing remote patients monitoring system with our proposed on real time bed monitoring system.

\section{CONCLUSION}

This paper proposes the novel framework for real time on bed medical services for patients suffering from pandemic and chronic diseases. The proposed framework shows how the real time patients monitoring done at different stages in the healthcare system such sensor node real time data processing and scheduling, real time data analytics at fog nodes, real time streaming of data using Kafka pipeline and real time data processing and analysis of large scale healthcare cloud data using Spark. The proposed framework helps in real time, reliable, fault tolerance and secure on bed patients care and monitoring. It also reduce the requirement of medical recourses including physical recourses, requirement of medical staffs, healthcare expenses and also reduce the death percentage of patients with pandemic and chronic diseases. In future the framework is implemented using cluster based cyber physical system for embedded sensor nodes using fog computing.

\section{REFERENCES}

A. Botta, WDDVPAP 2016, 'Integration of cloud computing and internet of things: a survey.', Futur. Gener. Comput. Syst., vol 56, pp. 684-700.

Abdulnabi, M 2017, 'A distributed framework for health information exchange using smartphone technologies.', J. Biomed. Inform. , vol 69, pp. 230-250.

Gu L, ZDGSBAXY 2017, ' Cost efficient resource management in fog computing supported medical cyber-physical system. ', IEEE Transactions on Emerging Topics in Computing, vol 5, no. 1, pp. 108-119.

Hussain, A,WR,DSAL,NM,AMM, 2015, 'Health and emergency-care platform for the elderly and disabled people in the Smart City',. J. Syst. Softw. , vol 110, pp. 
253-263.

Iqbal, SEA 2019, 'Real-time-based E-health systems: Design and implementation of a lightweight key management protocol for securing sensitive information of patients.', Health Technol. (Berl). 9(2):93-111, 2019., vol 9, no. 2, pp. 93-111.

K. I. Mohammed, 2019, ' Real Time Remote Health Monitoring System: A Review on Patients Prioritization for Multiple Chronic Diseases, Texonomy Analysis , Concern and solution procedure', Med System Springer, vol 43.

Kreps, J, Narkhede, N \&t Rao, JK 2011, 'A distributed messaging system for log processing. ', In Proceedings of the NetDB, Association for Computing Machinery, Athens, Greece.

M. Armbrust, RSXCLE 2015, 'Spark sql: relational data processing in spark,', International Conference on Management of Data , ACM, Melbourne,.

Mohsin, AE 2018, ' Real-time remote health monitoring systems using body sensor information and finger vein biometric verification: A multi-layer systematic review', J. Med. Syst.

Okura, T 2016, 'The importance of walking for control of blood pressure: Proof using a telemedicine system.', Telemed. e-Health , vol 22, no. 12, pp. 1019-1023.

R. Singhal and P. Singh 2014, 'Performance assurance model for applications on SPARK platform', Proceedings of the Technology Conference on Performance Evaluation and Benchmarking, , Springer, Munich.

Rahul Neware, US 2020, ' Fog Computing Architecture, Application and Security Issues', International Journal of Fog Computing, vol 3, pp. 75-105.

S. Gambhir, MK 2016, 'DWBAN: Dynamic priority based WBAN architecture for healthcaresystem', International Conference on Computing for Sustainable Global Development(INDIACom).

Zanjal, SV,ATGR 2016,' Medicine reminder and monitoring system for secure health using IoT', International Conference on Information Security \& Privacy, Procedia Comput. Sc, Nagpur. 